\title{
Género y cambio de actitudes ante el consumo de drogas. Los efectos del Programa de Competencia Familiar con adolescentes (12-14 años)*
}

\section{Gender and Attitude Change in Drug Abuse. Effects of the Family Competence Program with Adolescents (12-14 years)}

\author{
Carmen Orte \\ Universidad Islas Baleares, España \\ Lluís BALLESTER \\ Universidad Islas Baleares, España \\ JOAN AMER ${ }^{\mathrm{a}}$ \\ Universidad Islas Baleares, España \\ ORCID: http://orcid.org/0000-0001-7751-7110 \\ Marga Vives \\ Universidad Islas Baleares, España
}

a Autor de correspondencia. Correo electrónico: joan-amer@uib.cat

Para citar este artículo: Orte, C., Ballester, L., Amer, J., \& Vives, M. (2017). Género y cambio de actitudes ante el consumo de drogas. Los efectos del Programa de Competencia Familiar con adolescentes (12-14 años). Universitas Psychologica, 16(4), 1-12. https://do i.org/10.11144/Javeriana.upsy16-4.gcac

\section{RESUMEN}

Las actitudes en la adolescencia hacia el consumo de drogas son muy relevantes para entender el consumo real. Además, hay que incorporar perspectivas de explicación diferenciadas por género. En este estudio se analiza el cambio de actitudes en el largo plazo después de participar en un programa de prevención familiar basado en la evidencia, el Programa de Competencia Familiar (PCF). A partir de una muestra de 78 adolescentes que han participado en el programa, además de un grupo de control, se mide la evolución de las actitudes hacia el consumo mediante el Cuestionario de Actitudes hacia las Drogas (CAD), cuestionario validado y utilizado como referencia por el Plan Nacional Sobre Drogas español. Se llevan a cabo tres procesos de medición separados por género: al inicio de la aplicación del PCF, al final de la aplicación (Post 1) y un seguimiento a los 24 meses (Post 2). Los principales resultados indican que se consiguen diferencias significativas en la disminución de la disponibilidad hacia el consumo, y en en la reducción de las creencias erróneas al final del programa, aunque no a largo plazo, tanto en chicos como en chicas. En cuanto a las creencias correctas o protectoras, estas se mantienen consistentes a lo largo del tiempo en ambos sexos.

\section{Palabras clave}

adolescencia; prevención familiar; programa basado en la evidencia; actitudes hacia el consumo.

\section{ABSTRACT}

Attitudes towards drug abuse are relevant to understand real abuse. Furthermore, explanations by gender should be also incorporated. In the current study, it is analyzed the attitudinal change in the longterm after participating in an evidence-based family prevention program, the Family Competence Program (FCP). With a population sample of 
78 adolescents that have participated in the program, and a control group, it is measured the evolution of the attitudes towards consumption using the Drugs Attitudes Questionnaire, validated questionnaire and used as a reference by the Spanish National Plan Against Drugs. Three measurement processes are undertaken, differentiated by gender: at the beginning of the FCP application, at the end of the application (Post 1) and a 24-month follow-up (Post 2). Main results indicate that significant differences are achieved in availability for consumption and in wrong beliefs, at the end of the program, but not in the long term, both in girls and boys. Regarding protective beliefs, these are consistent along time in both genders.

Keywords

adolescence; family prevention; evidence-based program; substance use attitudes.

La adolescencia es una edad clave en el inicio del consumo de drogas, de hecho, como señala Becoña (2002), es en esta etapa evolutiva donde aparece un consumo predominante de drogas legales (alcohol y tabaco), es por ello que se necesitan análisis que incorporen los diferentes factores explicativos detrás de este inicio (Martins, Storr, Alexander, \& Chilcoat, 2008). Uno de estos factores es la conexión entre las actitudes hacia el consumo y el consumo real: diferentes estudios (De la Villa, Ovejero-Bernal, Castro, Rodríguez-Díaz, \& Sirvent-Ruiz, 2011; Kloos, Weller, Chan, \& Weller, 2009; Tonin, Burrow-Sanchez, Harrison, \& Kircher, 2008) determinan que las actitudes hacia las sustancias tienen un fuerte impacto en la posibilidad de consumir drogas.

Además, el consumo diferenciado por género presenta distintas pautas a lo largo de la adolescencia (Becoña, 2002). Por ejemplo, si bien el consumo de tabaco bajó en la población general, en el grupo de chicas adolescentes se incrementó en 2011 (Míguez \& Becoña, 2015). En cuanto al consumo de alcohol, según el Plan Nacional sobre Drogas (2015) aparece un repunte en los adolescentes, especialmente en la franja de los más jóvenes (14 y 15 años), donde la presencia de las chicas en consumo intensivo, borracheras, y binge drinking ha aumentado. Finalmente, la última Encuesta Estatal sobre Uso de Drogas en Estudiantes de Enseñanzas
Secundarias (Ministerio de Sanidad, Servicios Sociales e Igualdad, 2016) confirma que en mujeres el consumo de hipnosedantes sin receta $(6.8 \%)$ se ha duplicado en comparación con el grupo de hombres (3.8\%).

Chen y Jacobson (2012) exponen que las chicas tienen consumos más altos que los chicos en la primera adolescencia, mientras que los chicos muestran tasas de cambio más altas a lo largo del tiempo, con niveles más altos de consumo desde la mitad de la adolescencia hasta principio de la edad adulta. Según Kulis, Booth y Becerra (2016), durante la primera adolescencia, las chicas informan de más ocasiones en las que se les ofrecen sustancias. Por tanto, las problemáticas asociadas al consumo en la adolescencia también son diferentes según el género (Khan, Cleland, Scheidell, \& Berge, 2014). Estos autores indican, por ejemplo, que en el caso del consumo del alcohol, los problemas sociales asociados son mayores en el caso de las chicas, especialmente ligados a las relaciones sexuales y de pareja. Otro ejemplo es ver cómo la composición por género de los grupos de amigos incide en el consumo de sustancias (Jacobs, Goodson, Barry, \& McLeroy, 2016). Mrug, Borch y Cillesen (2011) encontraron que las chicas con mayor número de amigos chicos tenían mayor probabilidad de empezar a fumar. Giletta et al. (2012) exponen que las relaciones de amistad masculinas y femeninas son diferentes en estructura y contenido, y ello incide en maneras distintas en el consumo. Es por ello que, tal y como ya apuntaba Becoña en 2002, los programas de prevención específicos de género podrán disponer de resultados más favorables, especialmente en población de alto riesgo.

Siguiendo el modelo de ecología social sobre el consumo de drogas por género (Kumpfer, 2014; Kumpfer, Smith, \& Summerhays, 2008), el vínculo familiar y la supervisión de los padres tienen un impacto ligeramente mayor en las chicas en la elección de amigos que consumen o no consumen sustancias, de aquí la importancia para ellas de los programas de prevención familiar. Estos autores afirman que el autocontrol conductual y emocional tiene un papel un poco más grande en el consumo de 
drogas más adelante en los chicos, posiblemente porque los chicos parecen ser más propensos a las dificultades en esta área. Añaden que las chicas son más influenciadas por su rendimiento académico y la auto-eficacia que los chicos. Por último, exponen que la comunidad y el medio ambiente del barrio tienen una mayor influencia en chicos que en chicas.

En conjunto UNODC (2016) determina que hay factores de vulnerabilidad y capacidad de recuperación que parecen ser más o menos relevantes en las chicas que en los chicos y viceversa, y esto podría tener consecuencias para los programas y las políticas de prevención de drogas: I) las chicas están más influenciadas por la unión familiar y la supervisión de los padres, tanto en términos de vulnerabilidad, como en capacidad de recuperación; II) la influencia de los valores y las normas de los compañeros en relación al alcohol y el consumo de drogas en chicas y chicos parece ser igual de potente, pero expresado de manera diferente.

Como sugiere Kumpfer (2014), solo se evalúa una minoría de las estrategias de prevención de drogas, y de estas solo una pequeña parte ha llevado a cabo un análisis de su eficacia teniendo en cuenta las diferencias de género.

La prevención en el consumo de drogas puede ser efectiva si las intervenciones han sido evaluadas mediante investigaciones que siguen los estándares de eficacia basados en la evidencia (Gottfredson et al., 2015). Según estos autores, los programas basados en la evidencia pretenden dar a conocer las mejores pruebas científicas acerca de la eficacia de una intervención, con el objeto de ayudar en la toma de decisiones que el profesional tenga que adoptar en materia de política sanitaria, social y educativa: la evidencia se valida de acuerdo con una exhaustiva metodología de diseño del programa y de la investigación, las cuales permiten certificar si el programa se implementa de manera correcta y fidedigna, así como si se puede afirmar que los resultados se siguen de la aplicación del programa (SAMHSA, 2016).

El Programa de Competencia Familiar (PCF) es un programa de prevención, basado en la evidencia y validado con investigación cuasi- experimental y longitudinal (Orte, Ballester, \& March, 2013). Es la adaptación cultural española del Strengthening Families Program 6-11 de Kumpfer y Whiteside (2004), programa de prevención selectiva que ha demostrado su efectividad en la reducción de factores de riesgo asociados con el consumo de alcohol y drogas a través del fortalecimiento familiar, las competencias sociales de los adolescentes y las habilidades parentales (Kumpfer, Xie, \& O'Discroll, 2012). El PCF se trata de un programa multicomponente con sesiones para padres, hijos y familias, que trabaja con un enfoque cognitivo-emocional y técnicas de modificación de conducta. Contiene 14 sesiones, con refuerzo a los 6 meses y seguimiento a los 24 meses. Los objetivos generales del PCF son: I) mejora de las relaciones familiares; II) incremento de las competencias parentales; III) mejora de las competencias de los hijos (conocimiento, actitudes y conducta); IV) aumento de las habilidades sociales de los hijos; y $\mathrm{V}$ ) reducción o prevención del abuso de alcohol y otras drogas. Los objetivos específicos para los padres son el incremento $D E=$ la parentalidad positiva, la implicación parental, las habilidades parentales, la coparentalidad, la supervisión parental y la eficacia parental. En el caso de los hijos, los objetivos específicos son la mejora de las habilidades sociales, las relaciones interpersonales, las habilidades adaptativas escolares, la participación en la dinámica familiar, y de las actitudes preventivas al consumo. También en los hijos se persigue la reducción del consumo de drogas y de la agresividad.

Este programa, aplicado en unos 17 países diferentes (Kumpfer et al., 2012), presenta resultados positivos en cuanto a la prevención y/o reducción del consumo de sustancias; por ejemplo, Miller y Hendrie (2008) reportan que un $18 \%$ de adolescentes participantes en el SFP redujeron o no iniciaron el consumo de alcohol, un $15 \%$ de marihuana, un $11 \%$ de otras drogas, y un $7 \%$ de tabaco respecto a un grupo control (Magalhâes \& Kumpfer, 2015). Estas mismas autoras citan también los estudios de Brody, Chen, Kogan, Murry \& Brown (2010), Brody, 
Chen, Kogan et al. (2012) y Brody, Chen, Beach et al. (2014), relacionados con los efectos del SFP con la promoción de la salud mental, en concreto, la reducción de un 50\% de indicadores de depresión, ansiedad, abuso de sustancias, inicio de relaciones sexuales o conductas delictivas. La implementación del SFP en Portugal ha reportado también beneficios significativos en la comunicación familiar, la resiliencia o la cohesión familiar (Magalhâes \& Kumpfer, 2015).

A nivel nacional, el PCF ha demostrado resultados consistentes y de buena calidad en factores familiares referidos a padres, hijos y familia en general; tanto al finalizar el programa como en los seguimientos a 2 años (Orte et al., 2015) los procesos generados en la participación del programa han permitido mantener y mejorar a largo plazo la competencia familiar (Orte, Ballester, Pozo, \& Vives, 2017)

El objetivo del presente artículo es analizar la evolución de las actitudes hacia el consumo de drogas de los adolescentes participantes del PCF, que tienen entre 12 y 14 años en el momento del seguimiento longitudinal a los 24 meses, prestando especial atención a las diferencias por género.

Este estudio se ha diseñado para contrastar tres hipótesis relacionadas. Se trata de la diferenciación por género en los adolescentes de 12 a 16 años, de tres escalas básicas relativas a las actitudes hacia el consumo:

H1. Hay una significativamente mayor disponibilidad hacia el consumo entre los chicos que entre las chicas.

H2. Hay diferencias significativas sobre las creencias asociadas al riesgo de consumo entre chicos y entre chicas.

H3. Hay diferencias significativas sobre las creencias protectoras asociadas a la evitación del consumo entre chicos y entre chicas.

Como se puede ver, la primera hipótesis es de diferencia de grupos direccionales, es decir, establece el sentido de la diferenciación por género. La segunda y tercera hipótesis se establecen a partir de la diferenciación entre grupos, pero sin establecer una direccionalidad predefinida.

\section{Método}

Se trata de un diseño cuasi-experimental con grupo de control. Se realiza una evaluación longitudinal pre-post, así como un seguimiento a 24 meses, a 155 familias, con instrumentos validados para la población española. La aplicación y el estudio del PCF se realiza entre 2011 y 2015, con implementaciones en Proyecto Hombre (programa de rehabilitación del consumo de sustancias) en diferentes lugares de España y en servicios sociales de distintos municipios de las Islas Baleares. En el presente artículo se trabaja con los resultados de la evaluación de seguimiento de aquellos adolescentes que tienen entre 12 y 14 años a los 24 meses de la implementación del programa.

Para la recogida de datos en población menor de edad, los padres o representantes legales firmaron una autorización de participación en el programa, así como de obtención de datos a través de los instrumentos seleccionados para la evaluación del PCF, entre los que se incluye el CAD.

\section{Muestra}

En el análisis se han incluido solo los adolescentes que tenían entre 10 y 12 años al final de la aplicación, y que en el seguimiento a 24 meses tenían entre 12 y 14 años, participantes del PCF en implementaciones del Proyecto Hombre, y en Servicios Sociales anteriormente descritos. Es una muestra de 78 adolescentes, de los cuales 44 (58.4\%) son chicos y $34(43.6 \%)$ son chicas en los grupos experimentales tal y como se expone en la tabla 1: 
TABLA 1

Datos sociodemográficos de la muestra

\begin{tabular}{lccc}
\hline 12-14 años & Chicos & Chicas & TOTAL \\
\hline Proyecto Hombre & 21 & 14 & $\begin{array}{c}\mathbf{3 5} \\
(44.87 \%)\end{array}$ \\
Servicios Sociales A.P. & 23 & 20 & $\begin{array}{c}\mathbf{4 3} \\
(55.13 \%)\end{array}$ \\
\hline TOTAL & $\mathbf{4 4}$ & $\mathbf{3 4}$ & $\mathbf{7 8}$ \\
& $(58.41 \%)$ & $(43.59 \%)$ & $(100 \%)$ \\
\hline
\end{tabular}

Fuente: elaboración propia.

El grupo control tiene una dimensión de 21 adolescentes, de los cuales 8 (38.1\%) son chicos y $13(61.9 \%)$ son chicas. En cuanto a la dimensión del grupo control, hay que apuntar que a lo largo de la investigación sobre competencia familiar, se ha trabajado de manera agregada; solo en este artículo se hace análisis diferenciado por género.

Instrumento

La evaluación de las actitudes hacia el consumo de drogas se ha realizado mediante la aplicación del CAD, el Cuestionario de actitudes hacia el consumo de drogas, validado para población española (Macià, 1986), integrado por veinte ítems, en los que se indaga sobre el análisis de las dimensiones valorativa (creencias) y reactiva (inclinación para actuar: disposición para el consumo) de las actitudes tanto hacia el alcohol como hacia otras drogas. Por lo que respecta a las propiedades psicométricas, el valor de alpha estandarizado es moderadamente alto (0.8463); es decir, se trata de un cuestionario con muy buena consistencia interna.

Los veinte ítems se agrupan en tres escalas:

1. Disponibilidad hacia el consumo (CAD 1, 7, 10, 12, 14, 15, 17). Ejemplos:

1. ¿Probarías el tabaco por curiosidad? 10. ¿Comprarías tabaco para fumar?

15. ¿Consumirías drogas si tus amigos te animaran a ello?

2. Creencias erróneas RIESGO (CAD 2, $3,5,8,11,12,13,16,20)$.

Ejemplos:
8. ¿Crees que fumar tabaco te ayudaría a centrarte en tus estudios y a sentirte mejor?

11. ¿Piensas que tomar drogas de vez en cuando te ayudaría a superar tus problemas?

16. ¿Crees que el consumo de drogas te llevaría a vivir experiencias nuevas que te harían más feliz?

3. Creencias correctas PROTECTORAS (CAD 4, 6, 9, 18, 19).

Ejemplos:

6. ¿Estás convencido de que para tener una vida sana hay que pasar del alcohol?

18. ¿Rechazarías las drogas si te las ofrecieran?

19. Para mantenerse sano, ies mejor pasar de las drogas?

\section{Procedimiento}

Se estudia la trayectoria en las tres escalas de actitudes, por género, a lo largo de 24 meses. Los datos estadísticos se elaboraron por género, para el conjunto de la muestra. Las escalas construidas basadas en el cuestionario CAD se analizaron como variables cuantitativas. Las diferencias entre el grupo control y el grupo experimental se analizaron con el test para dos muestras independientes del grupo de tratamiento (experimental y control) y como factor entre sujetos.

Los cálculos de t de student (test paramétricos) o Mann-Whitney (test no paramétricos) se usaron según la normalidad de la distribución de los datos. Se comparó el resultado total para cada una de las tres escalas básicas en los tres momentos de recogida de datos (pre-test, posttest al final de la aplicación del PCF y seguimiento a 24 meses). La significación estadística fue aceptada en $p \leq 0.05$.

Se calculó el índice $Z$ de la prueba no paramétrica U de Mann-Whitney y su nivel de significación. Los resultados de la comparación pretest en los grupos experimental y control, a partir de la prueba, muestran valores significativamente similares. Los resultados de la 
comparación pretest y postest ( 1 y 2) muestran la eficacia comparada del programa $(\mathrm{PCF})$ en relación a las tres escalas de actitud estudiadas.

\section{Resultados}

En el caso de la primera escala, la disponibilidad hacia el consumo en el momento del punto de partida es más alta en los chicos $(4.5, \mathrm{DE}=$ 1.619) que en las chicas $(4.29, D E=1.503)$ (Figura 1 y Tabla 1). Al final de las 14 sesiones de trabajo, baja de manera consistente en ambos géneros (Chicos: 4.14, DE $=1.672$; y chicas: 3.86, $\mathrm{DE}=1.456)$, y después vuelve a subir bastante a los 24 meses (Chicos: 4.35, DE = 1.069; y chicas: $4.11, D E=1.617$ ), aunque no al nivel inicial evaluado. Se mantienen constantes las diferencias significativas entre ellos y ellas en los distintos momentos del análisis: en la valoración inicial $(p=0.05)$, en la valoración final del programa $(p=0.04)$ y en la valoración a los 24 meses (0.04). Por tanto, la recuperación en la disponibilidad hacia el consumo es más clara en los chicos, lo que afirma la primera hipótesis sobre mayor disponibilidad hacia el consumo entre chicos que entre chicas (H1).

Figura 1

Disponibilidad hacia el consumo

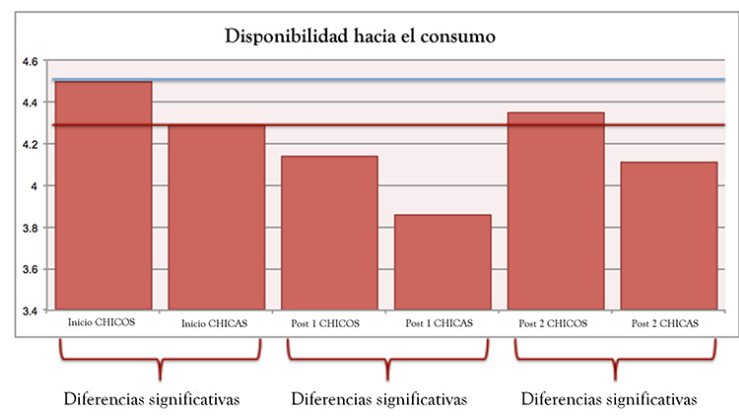

Fuente: elaboración propia.

En el caso de los chicos (Tabla 2), se consiguen diferencias significativas entre inicio y momento $1 /$ post $1(\mathrm{p}=0)$; se consigue que el cambio sea consistente. Se considera que hay mantenimiento de los efectos del programa, dado que, aunque la disponibilidad sube de manera importante al final de los 24 meses, no lo hace de manera estadísticamente significativa $(p$
$=0.154)$. En el caso de las chicas, también baja la disponibilidad hacia el consumo de manera significativa $(p=0.012)$ en el momento del postest y después vuelve a subir, sin que la diferencia en este segundo momento sea significativa $(p=0.539)$. Por tanto, se detecta un cierto mantenimiento de los efectos del programa, en cuanto a un relativo estancamiento y ligero descenso en su disponibilidad al consumo.

TABLA 2

Disponibilidad hacia el consumo. Chicos y chicas

\begin{tabular}{lcccc}
\hline GE 12-14 años & \multicolumn{2}{c}{ Chicos } & \multicolumn{2}{c}{ Chicas } \\
\hline Inicio PCF & $4.5(D E=1.619)$ & $4.29(D E=1.503)$ \\
Post 1 & $4.14(D E=1.672)$ & $3.86(D E=1.456)$ \\
Post 2 & $4.35(D E=1.069)$ & $4.11(D E=1.617)$ \\
\hline \multicolumn{3}{c}{ Chicos } & Chicas \\
\cline { 2 - 5 } & $t_{(43)}$ & $p$ & $t_{(33)}$ & $p$ \\
\hline Inicio PCF a Post 1 & 4.119 & 0 & 2.659 & 0.012 \\
Post 1 a Post 2 & -1.45 & 0.154 & -0.62 & 0.539 \\
\hline
\end{tabular}

Fuente: elaboración propia.

En el caso de la segunda escala de actitudes, relativas a las creencias erróneas (asociadas a riesgo), estas se encuentran prácticamente igualadas en el punto de partida en chicos (4.43, $D E=1.37)$ y chicas $(4.44, D E=1.617)$ (Figura 2 y Tabla 3). En ambos casos tiene lugar un descenso importante en el momento del Post 1 (chicos: $3.97, D E=1.171$; y chicas: $4.14, D E$ $=1.479$ ). La recuperación a los 24 meses (Post 2) es menor que en el caso de la disponibilidad hacia el consumo; por tanto, se mantienen mejor los efectos. En el análisis por género, las chicas presentan niveles más altos de creencias erróneas en el Post 1 y en el Post 2. La recuperación o aumento de los niveles de creencias erróneas es más consistente en el caso de las chicas. Las diferencias entre géneros no son significativas al inicio, pero después sí lo son en los momentos Post $1(p=0.05)$ y Post $2(p=0.04)$. 
Figura 2

Creencias erróneas (riesgo)

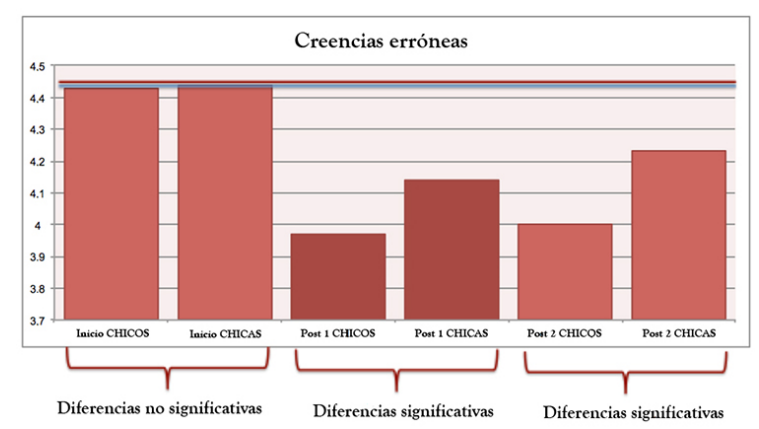

Fuente: elaboración propia.

Para los chicos (Tabla 3), las creencias erróneas bajan de manera consistente y se mantienen. El descenso del momento inicial al momento Post 1 es significativo $(p=0)$, mientras que el mantenimiento de Post 1 a Post 2, no es significativo $(p=0.864)$. Para las chicas, bajan de manera importante pero no tanto como en el caso de los chicos (momento Post 1, al final de la aplicación del PCF), y luego suben más que en el caso de los chicos. El descenso del inicio al Post 1 es significativo $(p=0)$. El ligero ascenso de Post 1 a Post 2 no es significativo $(p=0.609)$; así pues, se confirma la $\mathrm{H} 2$ planteada sobre las diferencias en creencias asociadas al riesgo entre chicos y chicas.

\section{TABLA 3}

Creencias erróneas (riesgo). Chicos y chicas

\begin{tabular}{lcccc}
\hline GE 12-14 años & \multicolumn{2}{c}{ Chicos } & \multicolumn{2}{c}{ Chicas } \\
\hline Inicio PCF & $4.43(D E=1.37)$ & $4.44(D E=1.617)$ \\
Post 1 & $3.97(D E=1.171)$ & $4.14(D E=1.479)$ \\
Post 2 & 4 & $(D E=1.2)$ & \multicolumn{2}{c}{$4.23(D E=1.371)$} \\
\hline & \multicolumn{2}{c}{ Chicos } & \multicolumn{2}{c}{ Chicas } \\
\cline { 2 - 5 } & $t_{(43)}$ & $p$ & $t_{(33)}$ & $p$ \\
\hline Inicio PCF a Post 1 & 4.131 & 0 & 2.726 & 0.01 \\
Post 1 a Post 2 & 0.172 & 0.864 & -0.517 & 0.609 \\
\hline
\end{tabular}

Fuente: elaboración propia.

En el caso de la tercera escala de actitudes, en las creencias correctas o protectoras (Figura $3 \mathrm{y}$ Tabla 4), el punto de partida es prácticamente igual entre chicos $(5.98, D E=0.769) \mathrm{y}$ chicas $(5.56, D E=1.377)$, con diferencia no significativa, y se mantiene aproximadamente igual a lo largo de los 24 meses. El mantenimiento es muy similar entre géneros y también el resultado final: las creencias correctas o protectoras son las que se mantienen de manera más consistente. En el Post 1 se produce un ligero aumento tanto en chicos $(6.9, \mathrm{DE}=0.603)$ como en chicas $(6.88, D E=0.327)$. En el Post 2 tiene lugar un pequeño descenso (chicos: 6.13, $D E=0.954$; chicas: $6.38, D E=0.985)$, que se acerca a los valores iniciales. No se encuentran diferencias significativas entre géneros. En este caso, pues, podemos descartar la hipótesis sobre las diferencias significativas entre sobre creencias protectoras asociadas a la evitación del consumo entre chicas y chicos $(\mathrm{H} 3)$.

\section{Figura 3}

Creencias correctas (protectoras)

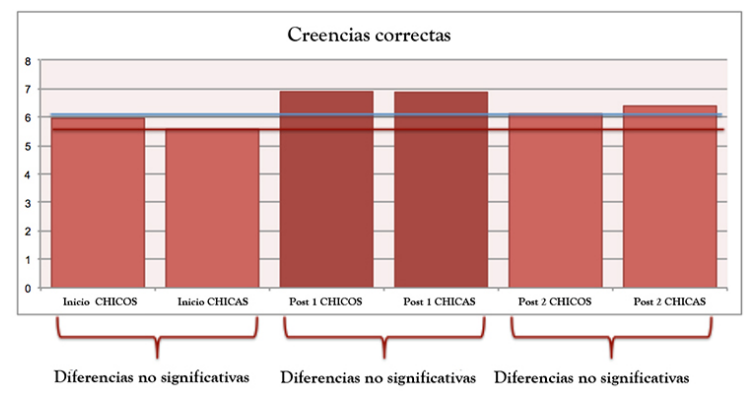

Fuente: elaboración propia.

En los chicos (Tabla 4), son significativos los cambios tanto del momento inicial al Post $1(p$ $=0)$, como del Post 1 al Post $2(p=0)$. En las chicas (Tabla 3), son también significativos los cambios entre los diferentes momentos: del inicio al Postest $1(p=0)$, y del Postest 1 al Postest $2(p$ $=0.005)$.

\section{TABLA 4}

Creencias correctas (protectoras). Chicos y chicas

\begin{tabular}{|c|c|c|c|c|}
\hline GE 12-14 años & \multicolumn{2}{|c|}{ Chicos } & \multicolumn{2}{|c|}{ Chicas } \\
\hline Inicio PCF & \multicolumn{2}{|c|}{$5.98(D E=0.769)$} & \multicolumn{2}{|c|}{$5.56(D E=1.377)$} \\
\hline Post 1 & \multicolumn{2}{|c|}{$6.9(D E=0.603)$} & \multicolumn{2}{|c|}{$6.88(D E=0.327)$} \\
\hline \multirow[t]{3}{*}{ Post 2} & \multicolumn{2}{|c|}{$6.13(D E=0.954)$} & \multicolumn{2}{|c|}{$6.38(D E=0.985)$} \\
\hline & \multicolumn{2}{|c|}{ Chicos } & \multicolumn{2}{|c|}{ Chicas } \\
\hline & $t_{(43)}$ & $p$ & $t_{(33)}$ & $p$ \\
\hline Inicio PCF a Post 1 & 13.22 & 0 & -5.416 & 0 \\
\hline Post 1 a Post 2 & 4.97 & 0 & 3.033 & 0.005 \\
\hline
\end{tabular}

Fuente: elaboración propia.

En la comparación entre los chicos y las chicas de los grupos experimentales, encontramos diferencias significativas en una serie de casos. Referentes a la disponibilidad hacia el consumo, las diferencias son significativas en los tres 
momentos (inicio, Post 1 y Post 2). Sobre las creencias erróneas, las diferencias son significativas en el Post 1 y en el Post 2. En cuanto a las creencias protectoras, no hay diferencias significativas en ninguno de los tres momentos, tal y como muestra la tabla 5.

\section{TABLA 5}

Comparación en grupo experimental entre chicos y chicas

\begin{tabular}{lccc}
\hline & \multicolumn{3}{c}{ Prueba t para la igualdad de medias } \\
\cline { 2 - 5 } & \multirow{2}{*}{$t$} & $g l$ & Sig. (bilateral) \\
\hline Disponibilidad hacia el consumo Inicio PCF-Pre & 1.576 & 76 & $0.0566^{*}$ \\
Creencias erróneas RIESGO Inicio PCF-Pre & -0.028 & 76 & 0.978 \\
Creencias correctas PROTECTORAS Inicio PCF-Pre & 1.715 & 76 & 0.0903 \\
\hline Disponibilidad hacia el consumo Final PCF-Post 1 & 1.799 & 76 & $0.0427^{*}$ \\
Creencias erróneas RIESGO Final PCF-Post 1 & -1.566 & 76 & $0.0573^{*}$ \\
Creencias correctas PROTECTORAS Final PCF-Post 1 & 0.233 & 76 & 0.8162 \\
\hline Disponibilidad hacia el consumo 24 meses-Post 2 & 1.745 & 76 & $0.0458^{*}$ \\
Creencias erróneas RIESGO 24 meses-Post 2 & -1.806 & 76 & $0.0423^{*}$ \\
Creencias correctas PROTECTORAS 24 meses-Post 2 & -1.113 & 76 & 0.2692 \\
\hline
\end{tabular}

Fuente: elaboración propia.

En cuanto a la comparación entre grupo experimental y grupo control (Tabla 6), las comprobaciones iniciales en la investigación sobre el PCF se hicieron para el conjunto, con garantía de resultados iniciales similares y con diferencias significativas o tendenciales en el post 1 y en el post 2 . En este trabajo presentamos de manera diferenciada por género para los objetivos del artículo, con alguna anomalía cuando se hace la diferencia desagregada.
TABLA 6

Comparación entre grupo experimental y grupo de control

\begin{tabular}{lcccc}
\hline CHICOS & $\begin{array}{c}\text { U de Mann- } \\
\text { Whitney }\end{array}$ & $\begin{array}{c}\text { Disponibilidad } \\
\text { hacia el } \\
\text { consumo }\end{array}$ & $\begin{array}{c}\text { Creencias } \\
\text { erróneas } \\
\text { RIESGO }\end{array}$ & $\begin{array}{c}\text { Creencias } \\
\text { correctas } \\
\text { PROTECTORAS }\end{array}$ \\
\hline Inicio PCF-Pre & $Z$ & -1.561 & -0.57 & -0.092 \\
Final PCF-Post1 & $p$ & 0.118 & 0.569 & 0.927 \\
& $Z$ & -1.723 & -1.764 & -3.106 \\
Seguimiento 24 meses-Post2 & $p$ & $0.085^{*}$ & $0.081^{*}$ & $0.002^{* *}$ \\
& $Z$ & -1.759 & -1.753 & -0.289 \\
& $p$ & $0.048^{* *}$ & $0.088^{*}$ & $0.773^{*}$ \\
\hline
\end{tabular}

Grupo experimental $(N=44)$

Grupo de control $(N=8)$

\begin{tabular}{lcccc}
\hline CHICAS & $\begin{array}{c}\text { U de Mann- } \\
\text { Whitney }\end{array}$ & $\begin{array}{c}\text { Disponibilidad } \\
\text { hacia el } \\
\text { consumo }\end{array}$ & $\begin{array}{c}\text { Creencias } \\
\text { erróneas } \\
\text { RIESGO }\end{array}$ & $\begin{array}{c}\text { Creencias } \\
\text { correctas } \\
\text { PROTECTORAS }\end{array}$ \\
\hline Inicio PCF-Pre & $Z$ & -3.37 & -1.381 & -0.694 \\
Final PCF-Post1 & $p$ & $0.001 * *$ & 0.167 & 0.488 \\
& $Z$ & -2.401 & -1.833 & -2.073 \\
Seguimiento 24 meses-Post2 & $P$ & $0.016^{* *}$ & $0.067^{*}$ & $0.038^{* *}$ \\
Grupo experimental $(N=34)$ & $p$ & -2.827 & -1.571 & -1.762 \\
Grupo de control $(N=13)$ & $0.005^{* *}$ & $0.072^{*}$ & $0.078^{*}$ \\
\multicolumn{5}{c}{$*$ Diferencias tendenciales $(<0.1)$} \\
$* *$ Diferencias significativas $(<0.05)$ \\
Fuente: elaboración propia.
\end{tabular}

\section{Discusión y conclusiones}

La etapa de la adolescencia puede definirse como una época de múltiples cambios en diferentes dimensiones (física, emocional, social, etc.), donde la búsqueda de uno mismo se configura como un elemento clave y donde los iguales adquieren una importancia relevante (Becoña, 2000). Al mismo tiempo, la variable género puede ayudar a explicar diferentes motivaciones específicas en las mujeres para iniciarse y continuar en el uso o en las consecuencias de uso (Romo, 2010) Tal y como señala Becoña (2002), el género es un factor significativo hacia la disponibilidad de droga, junto con otras variables como la presión de iguales, el abuso de sustancias en la familia, el estatus socioeconómico y la aculturación. En el Ministerio de Sanidad, Servicios Sociales e Igualdad (2016), desde 2010 disminuye la disponibilidad percibida de todas las drogas excepto para el alcohol, donde las chicas han aumentado de forma significativa su consumo.

La finalidad de la presente investigación fue estudiar el cambio de actitudes hacia el consumo de drogas a lo largo de la adolescencia, teniendo 
en cuenta la importancia de las actitudes como factor explicativo del consumo real (Kloos et al., 2009) e incorporando un análisis diferenciado por género.

$\mathrm{Si}$ bien es cierto que existe un reciente interés por la perspectiva de género en el diseño y evaluación de programas preventivos en consumo para adolescentes (Pozo, Orte \& Vives, 2016), la limitada investigación disponible sugiere que las estrategias de prevención de drogas no necesariamente tienen los mismos efectos en las chicas y en los chicos. Kumpfer (2014) añade que las estrategias basadas en la familia (como es el caso del Programa de Competencia Familiar) son eficaces generalmente para ambos, mientras que los programas basados en la comunidad y los basados en la escuela, a menudo no son tan eficaces para las chicas.

A corto plazo, se detectan resultados excelentes en la mejora de las actitudes: reducción de la disponibilidad de consumo, reducción de las creencias erróneas y aumento de las creencias protectoras. A largo plazo, la curiosidad y la disponibilidad para consumir se restablecen, especialmente entre los chicos. Las chicas muestran niveles menores de disponibilidad de consumo.

Las chicas muestran a largo plazo mayor restablecimiento de las creencias erróneas que los chicos, aunque sin alcanzar los niveles iniciales (pretest). En relación a estas creencias, se tendría que complementar el análisis con el estudio de los efectos de los mensajes del grupo de iguales y de los medios de comunicación de masas. En relación a las creencias protectoras, estas empiezan en un nivel alto, mejoran en el primer post-test y disminuyen después (aunque se mantienen más altas que la primera recogida de datos).

La bibliografía recogida en este artículo nos permite también debatir sobre los resultados obtenidos en las hipótesis planteadas, y ofrece alternativas como el hecho de que la información que se ofrece a los adolescentes, no es determinante para predecir consumos, o cómo determinadas creencias erróneas pueden afectar de forma más intensa a las adolescentes, por ejemplo la importancia de la imagen corporal.

En cuanto a las limitaciones, cabe decir que puede producirse deseabilidad social en las respuestas, sesgo que consiste en aportar respuestas socialmente aceptables a las pruebas de evaluación, para transmitir una imagen positiva. Puede deberse a varias cosas: desajuste psicológico, insinceridad consciente, necesidad de aceptación por parte de los investigadores u otras causas (Viladrich, 2005).

Referente a las líneas de futuro, se recomienda llevar a cabo sesiones de recordatorio a lo largo de los 24 meses posteriores a la finalización de la aplicación del PCF, para potenciar las mejoras observadas al final de la implementación; al mismo tiempo, será necesario también abordar las dinámicas de consumo actual, como las Nuevas Sustancias Psicoactivas (NSP), donde en Ministerio de Sanidad, Servicios Sociales e Igualdad (2016), se cita la alta percepción de disponibilidad en consumidores y la baja percepción del riesgo hacia estas, en donde los porcentajes de consumo corresponden a un $4.8 \%$ en hombres, y un 3.3.\% en mujeres, de 14 a 18 años.

En conjunto, el PCF demuestra buenas características protectoras en el contexto de los programas de prevención selectiva para adolescentes, como los demuestran los resultados en el cambio de actitudes. Además, en la línea de lo manifestado por Kumpfer (2014) y la UNODC (2016), el Programa de Competencia Familiar puede ser una buena opción para trabajar las actitudes hacia el consumo teniendo en cuenta las diferencias de género, puesto que se trabaja, de forma transversal, actitudes y/ estereotipos de género en la crianza de hijos, factor clave relacionado con el consumo (Becoña, 2002).

\section{Agradecimientos}

Agradecemos al Ministerio de Economía, Industria y Competitividad (MINECO), la Agencia Estatal de Investigación (AEI) y ea European Regional Development Funds (ERDF) 
por el apoyo al proyecto (MINECO/AEI/ERDF, $\mathrm{EU})$.

\section{Referencias}

Becoña, E. (2000). Los adolescentes y el consumo de drogas. Papeles del Psicólogo, 77, 25-32.

Becoña, E. (2002). Bases científicas de la prevención de las drogodependencias. Madrid: Delegación del Gobierno para el Plan Nacional Sobre Drogas.

Brody, G. H., Chen, Y.F., Kogan, S. M., Murry, V. M., \& Brown, A. C. (2010). Long-term effects of the Strong African American. Families program on youths' alcohol use. Journal of Consulting and Clinical Psychology, 78, 281-285.

Brody, G. H., Chen, Y.F., Kogan, S. M., Yu, T., Molgaard, V. K., Di Clemente, R. J., \& Wingood, G. M. (2012). Family-centered program to prevent substance use, conduct problems, and depressive symptoms in Black adolescents. Pediatrics, 129(1), 108 115.

Brody, G.H., Chen, Y.F., Beach, S.R., Kogan, S.M., Yu, T., Diclemente, R.J., Wingood, G.M., Windle, M., \& Philibert, R.A. (2014). Differential sensitivity to prevention programming: A dopaminergic polymorphism-enhanced prevention effect on protective parenting and adolescent substance use. Health Psych, 33(2), 182-191. doi10.1037/a0031253

Chen, P., \& Jacobson, K. C. (2012). Developmental trajectories of substance use from early adolescence to young adulthood: gender and racial/ethnnic differences. Journal of Adolescent Health, $50(2), 154-163$.

De la Villa-Jiménez, M., Ovejero-Bernal, A., Castro, A., Rodríguez-Díaz, F., \& SirventRuiz, C. (2011). Modificación de actitudes hacia el consumo de sustancias en adolescentes: seguimiento de las diferencias inter-género. International Journal of Clinical and Health Psychology, 11(2) 291-311.
Giletta, M., Scholte, R., Prinstein, M.J., Engels R.C.M.E., Rabaglietti, E., \& Burk, W. (2012). Friendship context matters: examining the domain specificity of alcohol and depression socialization among adolescents. Journal of Abnormal Child Psychology, 40 (7) 1027-1043.

Gottfredson, D.C., Cook, T.D., Gardner, F.E., Gorman-Smith, D., Howe, G.W., Sandler, I.N., \& Zafft, K.M. (2015). Standards of evidence for efficacy, effectiveness, and scale-up research in prevention science: next generation, Prevention Science, 16(7) 893-926.

Jacobs, W., Goodson, P., Barry, A., \& McLeroy, K. (2016). The Role of Gender in Adolescents' Social Networks and Alcohol, Tobacco, and Drug Use: A Systematic Review. Journal of School Health, 86(5) 322-333. https:// doi.org/10.1111/josh.12381

Khan, M., Cleland, C., Scheidell, J., \& Berge, A. (2014). Gender and racial/ ethnic differences in patterns of adolescent alcohol use and associations with adolescent and adult illicit drug use. The American Journal of Drug and Alcohol Abuse, 40(3) 213-224. https:// doi.org/10.3109/00952990.2014.892950.

Kloos, A., Weller, R. A., Chan, R., \& Weller, E. B. (2009). Gender differences in adolescent substance abuse. Current Psychiatry Reports, 11(2), 120-126.

Kulis, S., Booth, J., \& Becerra, D. (2016). DrugResistance Strategies of Early Adolescents in Mexico: Gender Differences in the Influence of Drug Offers and Relationship to the Offeror. Substance Use $\mathbb{E}$ Misuse, 51 (3) 370-382.

Kumpfer, K. (2014). Family-Based Intervention for the Prevention of Substance Abuse and Other Impulse Control Disorders in Girls. International Scholarly Research Notices. https://doi.org/10.1155/2014/308789

Kumpfer, K., Smith, P., \& Summerhays, J. F. (2008). A wake-up call to the prevention field: Are prevention programs for substance use effective for girls? Substance Use and Misuse, 43(8), 978-1001. 
Kumpfer, K., \& Whiteside, H. (2004). Strengthening Families Program 6-11 Years: Parent, Child and Family Skills Training Group Leaders Manuals. Salt Lake City, Lutra Group.

Kumpfer, K., Xie, J. \& O'Discroll, R. (2012). Effectiveness of a Culturally Adapted Strengthening Families Program 12-16 Years for Hogh-Risk Irish Families. Child Youth Care Forum, 41,143. https:// doi.org/10-1007/s10566-011-9168-0

Macià, D. (1986). Cuestionario de actitudes y Cuestionario de información. Método Conductual de Prevención de Drogodependencias. Valencia: Promolibro.

Magalhâes, C., \& Kumpfer, K. (2015). Prevention science: family evidence-based, adolescents and drug use. Modelos e projetos de inclusâo social. Porto: Escola Superior de Educaçâo de Viseu. 85-95. Recuperado de http://hdl.handle.net/1040 0.19/2888

Martins, S. S., Storr, C. L., Alexander, P. K., \& Chilcoat, H. D. (2008). Do adolescent ecstasy users have different attitudes towards drugs when compared to marijuana users? Drug and Alcohol Dependence, 94(13), 63-72.

Míguez, M.C. \& Becoña, E. (2015). ¿El consumo de cigarillos y alcohol se relaciona con el consumo de cannabis y el juego problema en adolescentes españoles? Adicciones, $27(1), 8-16$.

Miller, T.R., \& Hendrie, D. (2008). Substance abuse dollars and cents: A cost-benefit analysis. Center for Substance Abuse Prevention.

Ministerio de Sanidad, Servicios Sociales e Igualdad (2016). ESTUDES 2014/2015. Encuesta sobre uso de drogas en enseñanzas secundarias de España. Madrid: Ministerio de Sanidad, Servicios Sociales e Igualdad. Delegación del Gobierno para el Plan Nacional sobre Drogas.

Mrug, S., Borch, C., \& Cillessen, A. (2011). Other-sex friendships in late adolescence: risky associations for substance use and sexual debut? Journal of Youth and Adolescence, 40(7), 875-888. https:// doi.org/10.1007/s10964-010-9605-7

Plan Nacional Sobre Drogas. (2015). Consumo, percepciones y opiniones ante las drogas. Encuesta sobre Alcohol y Drogas en España (EDADES). Madrid: Ministerio de Sanidad, Servicios Sociales e Igualdad.

Pozo, R., Orte, C., \& Vives, M. (2016). Programas, intervenciones y prácticas efectivas en prevención de drogodependencias en mujeres jóvenes. Géneros. Multidisciplinary Journal of Gender Studies, 5(1) 859-886.

Orte, C., Ballester, L., \& March, M. (2013). The family competence approach. An experience of socio-educational work with families, Pedagogía Social. Revista Interuniversitaria, 21 (2) 3-27.

Orte, C., Ballester, L, March, M., Amer, J., Vives, M., \& Pozo, R. (2015). The Strengthening Families Programme in Spain: a long-term evaluation. Journal of Children's Services, 10(2), 101-119. https://doi.org/10.1108/ JCS-03-2013-0010

Orte, C.; Ballester, L.; Pozo, R. \& Vives, M. (2017). El mantenimiento de los cambios en un programa de prevención familiar basado en la evidencia. "Un estudio longitudinal de familias", Pedagogía social: revista interuniversitaria, 29(1), 113-127.

Romo, N. (2010). La mirada de género en el abordaje de los usos y abusos de drogas. Revista Española de Drogodependencias, 35(3), 269-272.

SAMHSA. (2016). SAMHSA's National Registry of Evidence-Based Programs and Practices (NREPP). Disponible en: http://n repp.samhsa.gov/

Tonin, S. L., Burrow-Sanchez, J. J., Harrison, R. S., \& Kircher, J. C. (2008). The influence of attitudes, acculturation, and gender on substance use for Mexican American middle school students. Addictive Behaviors, 33(7), 949-954.

UNODC, United Nations Office on Drugs and Crime. (2016). Guidelines on drug prevention and treatment for girls and women. Vienna: United Nations Office 
Carmen Orte, Lluís Ballester, Joan Amer, Et al.

on Drugs and Crime. Recuperado de https://www.unodc.org/documents/drug -prevention-and-treatment/unodc_2016_d rug_prevention_and_treatment_for_girls_and women E.pdf

Viladrich, M.C. (Coord.) (2005). Psicometría.

Barcelona: UOC.

\section{Notas}

* Artículo de investigación. 